\title{
Minimum rank of matrices described by a graph or pattern over the rational, real and complex numbers*
}

\author{
Avi Berman \\ Technion \\ Haifa 32000, Israel \\ Leslie Hogben \\ Department of Mathematics \\ Iowa State University \\ Ames, IA 50011, USA \\ lhogben@iastate.edu
}

Faculty of Mathematics

berman@techunix.technion.ac.il

\section{Shmuel Friedland}

Department of Mathematics, Statistics, and Computer Science University of Illinois at Chicago

Chicago, IL 60607-7045, USA

friedlan@uic.edu

Uriel G. Rothblum

Faculty of Industrial Engineering and Management Technion Haifa 32000, Israel

rothblum@ie.technion.ac.il

\section{Bryan Shader \\ Department of Mathematics \\ University of Wyoming \\ Laramie, WY 82071, USA \\ bshader@uwyo.edu}

Submitted: Apr 18, 2007; Accepted: Dec 22, 2007; Published: Feb 4, 2008

Mathematics Subject Classification: 05C50

\begin{abstract}
We use a technique based on matroids to construct two nonzero patterns $Z_{1}$ and $Z_{2}$ such that the minimum rank of matrices described by $Z_{1}$ is less over the complex numbers than over the real numbers, and the minimum rank of matrices described by $Z_{2}$ is less over the real numbers than over the rational numbers. The latter example provides a counterexample to a conjecture in [AHKLR] about rational realization of minimum rank of sign patterns. Using $Z_{1}$ and $Z_{2}$, we construct symmetric patterns, equivalent to graphs $G_{1}$ and $G_{2}$, with the analogous minimum rank properties. We also discuss issues of computational complexity related to minimum rank.
\end{abstract}

Keywords: minimum rank, graph, pattern, zero-nonzero pattern, field, matroid, symmetric matrix, matrix, real, rational, complex.

*This research began at the American Institute of Mathematics workshop, "Spectra of Families of Matrices described by Graphs, Digraphs, and Sign Patterns," and the authors thank AIM and NSF for their support. 


\section{Introduction}

The (real symmetric) minimum rank problem (for a graph) is to determine the minimum rank among real symmetric matrices whose zero-nonzero pattern of off-diagonal entries is described by a given (simple) graph $G$. The zero-nonzero pattern described by the graph has tremendous influence on minimum rank. For example, a matrix associated with a path on $n$ vertices $\left(P_{n}\right)$ is a symmetric tridiagonal matrix with nonzero sub- and superdiagonal, and thus has minimum rank $n-1$, whereas the complete graph on $n$ vertices $\left(K_{n}\right)$ has minimum rank 1 . For a discussion of the background of the minimum rank problem (and an extensive bibliography), see [FH].

Much of the work on the minimum rank problem has focused on real symmetric matrices, but symmetric matrices over other fields have also been studied (see [BHL]). While examples of differences in minimum rank over different fields are known, these examples involve fields of different characteristic or size. We use a technique based on matroids to construct two zero-nonzero patterns $C_{S_{1}}$ and $C_{S_{2}}$ such that the minimum rank of matrices described by $C_{S_{1}}$ is less over the complex numbers than over the real numbers ${ }^{1}$, and the minimum rank of matrices described by $C_{S_{2}}$ is less over the real numbers than over the rational numbers. The pattern $C_{S_{2}}$ immediately provides a counterexample to a conjecture in [AHKLR] about rational realization of minimum rank of sign patterns. We then use $C_{S_{1}}$ and $C_{S_{2}}$ to construct symmetric patterns, equivalent to graphs $G_{1}$ and $G_{2}$, with the analogous minimum rank properties. All graphs discussed in this paper are simple, meaning no loops or multiple edges. The order of a graph $G$, denoted $|G|$, is the number of vertices of $G$.

For a symmetric $n \times n$ matrix $A$ over a field $F$, the graph of $A$, denoted $\mathcal{G}(A)$, is the graph with vertices $\{1, \ldots, n\}$ and edges $\left\{\{i, j\} \mid a_{i j} \neq 0\right.$ and $\left.i \neq j\right\}$. Note that the diagonal of $A$ is ignored in determining $\mathcal{G}(A)$. The set of symmetric matrices of the graph $G$ over the field $F$ is

$$
\mathcal{S}_{G}^{F}=\left\{A \in F^{n \times n}: A^{T}=A \text { and } \mathcal{G}(A)=G\right\} .
$$

Since we will need to consider non-symmetric matrices, as well as matrices over the rational and complex numbers, we adopt the perspective that we are finding the minimum of the ranks of the matrices in a given family $\mathcal{F}$ of matrices, and define

$$
\operatorname{mr}(\mathcal{F})=\min \{\operatorname{rank}(A): A \in \mathcal{F}\}
$$

Note that what we are denoting by $\operatorname{mr}\left(\mathcal{S}_{G}^{\mathbb{R}}\right)$ is commonly denoted by $\operatorname{mr}(G)$ in papers that study only the minimum rank of the real symmetric matrices described by a graph, and $\operatorname{mr}\left(\mathcal{S}_{G}^{F}\right)$ is sometimes denoted by $\operatorname{mr}(F, G)$ or $\operatorname{mr}^{F}(G)$.

Clearly $\operatorname{mr}\left(\mathcal{S}_{G}^{\mathbb{Q}}\right) \geq \operatorname{mr}\left(\mathcal{S}_{G}^{\mathbb{R}}\right) \geq \operatorname{mr}\left(\mathcal{S}_{G}^{\mathbb{C}}\right)$, but in all previously known examples, including all graphs having minimum rank less than 3 , the minimum rank was the same for all fields of characteristic zero [BHL]. Using the notation just introduced, in Section 3 we show

\footnotetext{
${ }^{1}$ We thank Chris Godsil and Jim Oxley for providing references to relevant papers on matroids. A good general reference on matroids is $[\mathrm{O}]$.
} 
that $\operatorname{mr}\left(\mathcal{S}_{G_{1}}^{\mathbb{R}}\right)>\operatorname{mr}\left(\mathcal{S}_{G_{1}}^{\mathbb{C}}\right)$ and $\operatorname{mr}\left(\mathcal{S}_{G_{2}}^{\mathbb{Q}}\right)>\operatorname{mr}\left(\mathcal{S}_{G_{2}}^{\mathbb{R}}\right)$. However, these examples are quite large (the orders are 75 and 181, respectively). First we show that for very small graphs (order $\leq 6$ ), all these minimum ranks are equal.

A cut-vertex of a connected graph is a vertex whose deletion disconnects $G$. In $[\mathrm{BFH}]$ it was shown that if $G$ has a cut-vertex, the problem of computing the minimum rank of $G$ can be reduced to computing minimum ranks of certain subgraphs. Specifically, let $v$ be a cut-vertex of $G$. For $i=1, \ldots, h$, let $W_{i}$ be the vertices of the $i$ th component of $G-v$ and let $G_{i}$ be the subgraph induced by $\{v\} \cup W_{i}$. Then $r_{v}(G)=\min \left\{\sum_{1}^{h} r_{v}\left(G_{i}\right), 2\right\}$, where $r_{v}(G)=\operatorname{mr}(G)-\operatorname{mr}(G-v)$ is called the rank-spread of $G$ at vertex $v$. Thus

$$
\operatorname{mr}(G)=\sum_{1}^{h} \operatorname{mr}\left(G_{i}-v\right)+\min \left\{\sum_{1}^{h} r_{v}\left(G_{i}\right), 2\right\} .
$$

Wayne Barrett has observed that the proof remains valid over any field. Hence we have the following.

Observation 1.1. If the minimum rank of $H$ is independent of field for all $H$ such that $|H|<|G|$ and $G$ has a cut-vertex, then the minimum rank of $G$ is independent of field.

Throughout this paper. $\mathbb{F}$ denotes a field of characteristic 0 , and $\mathbb{F}^{n}$ denotes the set of $n$ by 1 vectors with entries in $\mathbb{F}$.

A graph is 2-connected if its order is at least 3 and it has no cut-vertex. A linear 2 -tree is a 2-connected graph $G$ that can be embedded in the plane such that the graph obtained from the dual of $G$ after deleting the vertex corresponding to the infinite face is a path. Equivalently, a linear 2-tree is a "path" of cycles built up one cycle at a time by identifying an edge of a new cycle with an edge (that has a vertex of degree 2) of the most recently added cycle. In $[\mathrm{HH}]$ it is established that for a 2-connected graph $G$, $\operatorname{mr}\left(\mathcal{S}_{G}^{\mathbb{R}}\right)=|G|-2$ if and only if $G$ is a linear 2-tree, but the proof is specific to the real numbers. In [JLS], a complete characterization of graphs having minimum rank $|G|-2$ over infinite fields is given, and as a consequence it is shown that for any infinite field $\mathbb{F}$, $\operatorname{mr}\left(\mathcal{S}_{G}^{\mathbb{F}}\right)=|G|-2$ if and only if $G$ is a linear 2-tree. (Note that in [JLS] what we call a linear 2-tree is called a linear singly edge-articulated cycle graph or LSEAC graph.)

Proposition 1.2. Let $G$ be a connected graph such that $|G| \leq 6$ and let $\mathbb{F}$ be a field of characteristic 0. Then $\operatorname{mr}\left(\mathcal{S}_{G}^{\mathbb{F}}\right)=\operatorname{mr}\left(\mathcal{S}_{G}^{\mathbb{R}}\right)$. In particular, $\operatorname{mr}\left(\mathcal{S}_{G}^{\mathbb{Q}}\right)=\operatorname{mr}\left(\mathcal{S}_{G}^{\mathbb{R}}\right)=\operatorname{mr}\left(\mathcal{S}_{G}^{\mathbb{C}}\right)$ for any graph $G$ such that $|G| \leq 6$.

Proof. The result is clear if $|G|=1,2$. In general, $\operatorname{mr}\left(\mathcal{S}_{G}^{\mathbb{F}}\right)=1$ if and only if $G$ is a complete graph, and $\operatorname{mr}\left(\mathcal{S}_{G}^{\mathbb{F}}\right)=|G|-1$ if and only if $G$ is a path. The latter statement is a consequence of Fiedler's Tridiagonal Matrix Theorem (proved over the real numbers in $[\mathrm{F}]$; the proof in $[\mathrm{RS}]$ is valid for any field of characteristic 0$)$. This establishes the result for $|G|=3,4$. From [BHL], if $|G|=5, \operatorname{mr}\left(\mathcal{S}_{G}^{\mathbb{F}}\right)=2$ if and only if $G$ is not $K_{5}$, not Dart, not $\ltimes$, and $G$ does not contain $P_{4}$ as an induced subgraph (see Figure 1). For $|G|=5$ this is sufficient to establish the result, since for $|G|=5$, graphs having minimum rank 
3 over $F$ are precisely those not having minimum rank 1, 2, or 4 . In [HH] and [JLS] it is shown that for graphs $G$ without cut-vertices, $\operatorname{mr}\left(\mathcal{S}_{G}^{\mathbb{F}}\right)=|G|-2$ if and only if $G$ is a linear 2-tree. Together with the fact that the result is true for $|G| \leq 5$ and Observation 1.1, this establishes the result for $|G|=6$.

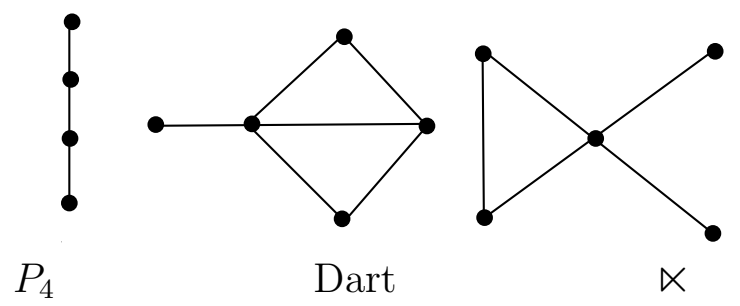

Figure 1: Some forbidden induced subgraphs for $\operatorname{mr}\left(\mathcal{S}_{G}^{\mathbb{F}}\right) \leq 2$

Obviously Proposition 1.2 can be applied to conclude that there is no difference in minimum rank over fields of characteristic zero for graphs having each connected component of order 6 or less, and can be combined with Observation 1.1 to to show that many additional small graphs have no difference in minimum rank over fields of characteristic zero.

There is a graph of order 6 for which the minimum rank over $\mathbb{Z}_{2}$ differs from the minimum rank over $\mathbb{R}$.

Example 1.3. Let $K_{3} \square K_{2}$ be the graph constructed from two copies of $K_{3}$ joined by a complete matching; $K_{3} \square K_{2}$ is shown in Figure 2. Then $\operatorname{mr}\left(\mathcal{S}_{K_{3}}^{\mathbb{R}} \square K_{2}\right)=3$ since $K_{3} \square K_{2}$ has an induced $P_{4}$ but is not a linear 2-tree (in fact, the block matrix $\left[\begin{array}{cc}J-I & I \\ I & (J-I)^{-1}\end{array}\right]$, where $I$ is the identity matrix and $J$ is the all ones matrix, has rank 3 ).

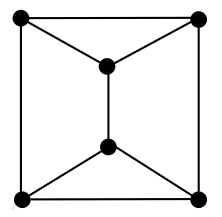

Figure 2: The graph $K_{3} \square K_{2}$

With appropriate ordering of the vertices, any matrix in $\mathcal{S}^{\mathbb{Z}_{2}}\left(K_{3} \square K_{2}\right)$ is of the form

$$
\left[\begin{array}{cccccc}
d_{1} & 1 & 1 & 1 & 0 & 0 \\
1 & d_{2} & 1 & 0 & 1 & 0 \\
1 & 1 & d_{3} & 0 & 0 & 1 \\
1 & 0 & 0 & d_{4} & 1 & 1 \\
0 & 1 & 0 & 1 & d_{5} & 1 \\
0 & 0 & 1 & 1 & 1 & d_{6}
\end{array}\right]
$$


and computation using all 64 possible $\left(d_{1}, \ldots, d_{6}\right)$ shows the rank is at least 4.

In order to construct our examples of graphs where the minimum rank differs over $\mathbb{R}$ and $\mathbb{C}$ or over $\mathbb{R}$ and $\mathbb{Q}$, we will first need to construct examples over non-symmetric nonzero patterns. A nonzero pattern $Z=\left[z_{i j}\right]$ is a matrix whose entries $z_{i j}$ are elements of $\{*, 0\}$. Given a pattern $Z=\left[z_{i j}\right]$, we let $\mathcal{M}_{Z}^{F}$ denote the set of all matrices $A=\left[a_{i j}\right]$ over $F$ such that $a_{i j} \neq 0$ if and only if $z_{i j}=*$. A realization of $Z$ over $F$ is a matrix in $\mathcal{M}_{Z}^{F}$. Note that (unlike the set of symmetric matrices described by a graph), here the diagonal is constrained by the zero-nonzero pattern.

\section{Minimum ranks of patterns over the rational, real and complex numbers}

Let $V$ be an $n$ by $k$ matrix over $\mathbb{F}$. We denote the nullspace of $V,\left\{w \in \mathbb{F}^{k}: V w=0\right\}$, by $\mathrm{NS}(V)$, and the left nullspace of $V,\left\{w \in \mathbb{F}^{n}: w^{T} V=0\right\}$, by $\operatorname{LNS}(V)$. Throughout most of this section, the of rank of $V$ will be $k$; in this case, $\operatorname{dim}(\operatorname{LNS}(V))=n-\operatorname{rank} V=n-k$. For an $m$ by $n$ matrix $A$ over $\mathbb{F}$, we denote the row space of $A$ (the subspace of $\mathbb{F}^{n}$ spanned by the rows of $A$ ) by $\operatorname{row}(A)$.

A cycle of $V$ is a subset $\alpha$ of $\{1,2, \ldots, n\}$ such that the rows of $V$ indexed by $\alpha$ are linearly dependent and each proper subcollection of these columns is linearly independent. Let $\vec{\alpha}$ denote the 1 by $n$ pattern obtained from $\alpha$ by placing a $*$ in position $j$ when $j \in \alpha$, and a 0 in position $j$ otherwise. A cycle matrix $C_{V}$ of $V$ is a matrix whose rows are the patterns $\vec{\alpha}$ as $\alpha$ runs over the cycles of $V$. Note that we don't prescribe the ordering of the rows of $C_{V}$. Thus $V$ has many cycle matrices, but they are all obtained from a single cycle matrix by permutation of rows.

Lemma 2.1. Let $V$ be an $n$ by $k$ matrix of rank $k$ with entries from $\mathbb{F}$, and let $C_{V}$ be a cycle matrix of $V$. Also, let $\alpha$ be the set of indices of a collection of linearly independent rows of $V$. Then there exists a subset $\beta$ of row indices and a subset $\gamma$ of column indices such that $\alpha \cap \gamma=\emptyset$ and $C_{V}[\beta, \gamma]$ is an $(n-k)$ by $(n-k)$ matrix whose rows can be permuted to the matrix

$$
\left[\begin{array}{ccccc}
* & 0 & 0 & \cdots & 0 \\
0 & * & 0 & \cdots & 0 \\
0 & 0 & * & \ddots & 0 \\
\vdots & \vdots & \ddots & \ddots & \vdots \\
0 & 0 & \cdots & 0 & *
\end{array}\right]
$$

Proof. Since $V$ has rank $k$, we may assume without loss of generality that $\alpha$ is $\{1,2, \ldots, k\}$. For each $j \in\{k+1, \ldots, n\}$, rows $1,2, \ldots, k, j$ of $V$ are linearly dependent, and thus there is a cycle of $V$ containing $j$ and contained in $\{1,2, \ldots, k, j\}$. Hence, there is a row of $C_{V}$ with $\mathrm{a} *$ in column $j$, and 0 s in all positions $\ell$ with $\ell>k$ and $\ell \neq j$. The result now follows. 
Lemma 2.2. Let $V$ be an $n$ by $k$ matrix of rank $k$ with entries from the field $\mathbb{F}$, and let $C_{V}$ be a cycle matrix of $V$. Then $\operatorname{mr}\left(\mathcal{M}_{C_{V}}^{\mathbb{T}}\right)=n-k$.

Proof. By Lemma 2.1, $\operatorname{mr}\left(\mathcal{M}_{C_{V}}^{\mathbb{F}}\right) \geq n-k$. For each row $\alpha$ of $C_{V}$ there is a realization of $\alpha$ that belongs to $\operatorname{LNS}(V)$. Hence, there is a realization $A \in \mathcal{M}_{C_{V}}^{\mathbb{R}}$ such that $A V=O$. Thus, $\operatorname{mr}\left(\mathcal{M}_{C_{V}}^{\mathbb{F}}\right) \leq \operatorname{rank}(A) \leq n-\operatorname{rank}(V)=n-k$.

In his early work on matroids $[\mathrm{M}]$, Saunders MacLane gave examples of matroids that can be represented over the complex number but not the real numbers and over the real numbers but not the rational numbers. We use these ideas to construct two matrices, and from these matrices, patterns that have differing minimum ranks. We begin with the example that distinguishes the complex numbers from the real numbers. Let

$$
S_{1}=\left[\begin{array}{ccc}
1 & 0 & 0 \\
0 & 1 & 0 \\
1 & 1 & 0 \\
1 & \omega+1 & \omega \\
0 & 0 & 1 \\
1 & \omega+1 & \omega+1 \\
1 & 1 & \omega+1 \\
0 & 1 & 1 \\
1 & 0 & \omega
\end{array}\right]
$$

where $\omega=\frac{-1+\sqrt{3} i}{2}$.

It is not difficult to verify that the cycles of $S_{1}$ correspond to the lines and 4-sets of points in general position of $\mathrm{AG}(2,3)$, the affine plane of order 3, as labeled in Figure 3. There are 123 -cycles (see Figure 3). Since there are $\left(\begin{array}{l}9 \\ 4\end{array}\right)$ 4-element subsets, and each 3 -cycle excludes 6 of these, there are $126-(6)(12)=54$-cycles and thus a total of 66 cycles of $S_{1}$.

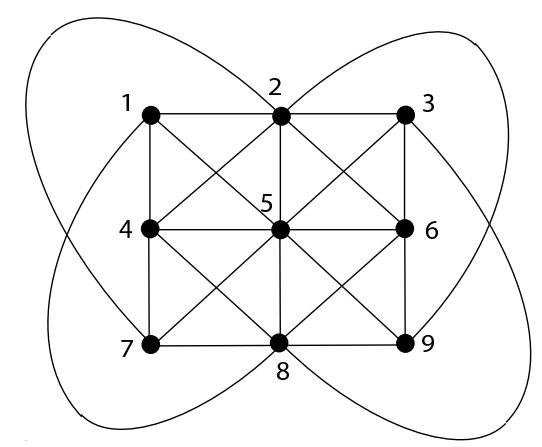

Figure 3: Diagram of $\mathrm{AG}(2,3)$ for $S_{1}$

We shall make use of several known results, which are a matrix theoretic restatement of MacLane's results on matroids. 
Theorem 2.3. There is no real matrix $T$ such that $C_{T}=C_{S_{1}}$.

Proof. Suppose to the contrary that there exists a 9 by $\ell$ real matrix $W=\left[w_{i j}\right]$ of rank $\ell$ whose cycle matrix is $C_{S_{1}}$. Since every cycle of $S_{1}$ has at least 3 elements, each pair of rows of $W$ are linearly independent. Since every set of 4 rows of $S_{1}$ is linearly dependent, so is every set of 4 rows of $W$. Hence $W$ has rank at most 3 and $\ell \leq 3$. Rows 1,2 and 5 of $S_{1}$ are linearly independent. Thus no cycle of $S_{1}$ (and hence of $W$ ) is contained in $\{1,2,5\}$. Therefore, rows $1,2,5$ of $W$ are linearly independent. Therefore, $W$ has rank 3 , that is, $\ell=3$.

Note that post-multiplying $W$ by an invertible (real) matrix, or pre-multiplying $W$ by an invertible (real) diagonal matrix does not change its cycle matrix. Thus, we may assume without loss of generality that the leftmost nonzero entry in each row of $W$ is a 1 and that

$$
W[\{1,2,5\},:]=I_{3} .
$$

Because $\{1,2,3\}$ is a cycle, and each pair of columns of $W$ is linearly independent, we have that $w_{31} \neq 0, w_{32} \neq 0$ and $w_{33}=0$. Thus, by scaling columns and then rows, we may assume without loss of generality that

$$
W[\{1,2,3,5\},:]=\left[\begin{array}{lll}
1 & 0 & 0 \\
0 & 1 & 0 \\
1 & 1 & 0 \\
0 & 0 & 1
\end{array}\right] .
$$

Similarly, using that $\{2,5,8\}$ is a cycle of $S_{1}$, we conclude that without loss of generality row 8 of $W$ is

$$
\left[\begin{array}{lll}
0 & 1 & 1
\end{array}\right] \text {. }
$$

Using that $\{1,5,9\}$ is a cycle, we see that row 9 of $W$ is

$$
\left[\begin{array}{lll}
1 & 0 & a
\end{array}\right]
$$

for some nonzero real number $a$.

Next use that $\{3,5,7\}$ is a cycle to conclude that row 7 of $W$ is

$$
\left[\begin{array}{lll}
1 & 1 & b
\end{array}\right]
$$

for some nonzero real number $b$.

Next use that $\{1,6,8\}$ is a cycle to conclude that row 6 of $W$ has the form

$$
\left[\begin{array}{lll}
1 & c & c
\end{array}\right]
$$

for some nonzero real number $c$. 
Thus, we have that $W$ has the form

$$
\left[\begin{array}{lll}
1 & 0 & 0 \\
0 & 1 & 0 \\
1 & 1 & 0 \\
x & y & z \\
0 & 0 & 1 \\
1 & c & c \\
1 & 1 & b \\
0 & 1 & 1 \\
1 & 0 & a
\end{array}\right]
$$

for some nonzero real numbers, $a, b, c$ and real numbers $x, y, z$.

Since $\{7,8,9\}$ is a cycle,

$$
0=\operatorname{det}\left[\begin{array}{lll}
1 & 1 & b \\
0 & 1 & 1 \\
1 & 0 & a
\end{array}\right]=a+1-b
$$

Since $\{3,6,9\}$ is a cycle,

$$
0=\operatorname{det}\left[\begin{array}{lll}
1 & 1 & 0 \\
1 & c & c \\
1 & 0 & a
\end{array}\right]=a c+c-a
$$

Since $\{2,6,7\}$ is a cycle,

$$
0=\operatorname{det}\left[\begin{array}{lll}
0 & 1 & 0 \\
1 & c & c \\
1 & 1 & b
\end{array}\right]=c-b
$$

These equations lead to $b=a+1, a c+c-a=0$, and $c=b$. Thus, $c=a+1$, and substitution into the second equation gives: $a^{2}+a+1=0$. Therefore, $a=\frac{-1 \pm \sqrt{-3}}{2}$, which contradicts the fact that $W$ is a real matrix.

Therefore, there is no real matrix whose cycle matrix is $C_{S_{1}}$.

Corollary 2.4. $\operatorname{mr}\left(\mathcal{M}_{C_{S_{1}}}^{\mathbb{R}}\right)=7>6=\operatorname{mr}\left(\mathcal{M}_{C_{S_{1}}}^{\mathbb{C}}\right)$.

Proof. By Lemma 2.2, $\operatorname{mr}\left(\mathcal{M}_{C_{S_{1}}}^{\mathbb{C}}\right)=6$.

Let $A$ be a real realization of $C_{S_{1}}$ of minimum rank. We claim that $\operatorname{rank}(A) \geq 7$. Suppose to the contrary that $\operatorname{rank}(A) \leq 6$. Let $W$ be a real matrix whose columns form a basis for the nullspace of $A$. By Lemma 2.1, $C_{S_{1}}$ contains a submatrix that is a 6 by 6 permutation matrix. Thus, $\operatorname{rank}(A)=6$ (and so $W$ has 3 columns). Note that since $\operatorname{dim} \operatorname{row}(A)=\operatorname{rank}(A)=6=9-\operatorname{rank}(W), \operatorname{row}(A)=\operatorname{LNS}(W)$

Let $\alpha$ be a collection of row indices such that the set of rows of $S_{1}$ indexed by $\alpha$ is linearly independent. By Lemma 2.1, $6 \leq \operatorname{rank}(A[:, \bar{\alpha}])$. The existence of a nonzero vector 
$v \in \operatorname{row}(A)$ whose support is contained in $\alpha$ leads to the contradiction $6=\operatorname{rank}(A) \geq$ $1+\operatorname{rank}(A[:, \bar{\alpha}]) \geq 1+6=7$. Thus, the row space of $A$ contains no nonzero vector whose support is contained in $\alpha$. Since $\operatorname{row}(A)=\operatorname{LNS}(W)$, the set of rows of $W$ indexed by $\alpha$ is linearly independent. We have shown: whenever a collection of rows of $S_{1}$ is linearly independent, the corresponding collection of rows of $W$ is also linearly independent (or equivalently, if a collection of rows of $W$ is linearly dependent, then the corresponding collection of rows of $S_{1}$ is also linearly dependent). In particular, no pair of rows of $W$ is linearly dependent.

Let $\alpha$ be a cycle of $W$ of size 3 . Then by the preceding observation the rows of $S_{1}$ indexed by $\alpha$ are linearly dependent, and since each pair of rows of $S_{1}$ is linearly independent, $\alpha$ is a cycle of $S_{1}$ of size 3 .

Let $\beta$ be a cycle of $S_{1}$ of size 3 . Then $A$ contains a nonzero row whose support is $\beta$, and hence the rows of $W$ indexed by $\beta$ are linearly dependent. Since each pair of rows of $W$ is linearly independent, $\beta$ is a cycle of $W$ of size 3 .

We have shown that $V$ and $W$ have the same cycles of size 3 . The cycles of $W$ (respectively, $S_{1}$ ) of size 4 are precisely the 4 -sets which contain no cycle of size 3 . Thus, the cycles of $W$ and $S_{1}$ of size 4 are equal. Since both $W$ and $S_{1}$ have rank 3 , it follows that $W$ and $S_{1}$ have the same cycles. This contradicts Theorem 2.3.

Therefore, $\operatorname{mr}\left(\mathcal{M}_{C_{S_{1}}}^{\mathbb{R}}\right) \geq 7>6=\operatorname{mr}\left(\mathcal{M}_{C_{S_{1}}}^{\mathbb{C}}\right)$.

To see that $\operatorname{mr}\left(\mathcal{M}_{C_{S_{1}}}^{\mathbb{R}}\right)=7$, consider the 9 by 2 real matrix $X$ whose $j$ th row is $[1, j]$. Clearly, every 2 by 2 submatrix of $X$ is invertible, and hence for each 1 by 9 pattern with 3 or more nonzeros there is a realization that belongs to the left nullspace of $X$. Therefore, there is a realization of $\mathcal{M}_{C_{S_{1}}}^{\mathbb{R}}$ of rank at most (and hence exactly) 7 .

Note that in the proof of Theorem 2.3, no cycle of $S_{1}$ containing 4 is used. It follows that there is no real matrix whose cycles are the same as those of $S_{1}[\overline{\{4\}},:]$. As the points of $\operatorname{AG}(3,2)$ are interchangeable, there is no real matrix whose cycles are the same as those of $S_{1}[\overline{\{j\}},:]$ for each $j$. This observation and an argument similar to that of Corollary 2.4 prove the following.

Corollary 2.5. Let $S$ be a pattern obtained from $S_{1}$ by deleting a row. Then

$$
\operatorname{mr}\left(\mathcal{M}_{C_{S}}^{\mathbb{R}}\right)=6>5=\operatorname{mr}\left(\mathcal{M}_{C_{S}}^{\mathbb{C}}\right) .
$$

We now construct an example that distinguishes the rational numbers from the real 
numbers. Let

$$
S_{2}=\left[\begin{array}{ccc}
1 & \frac{1}{2}+\frac{\sqrt{5}}{2} & 0 \\
1 & 1 & 1 \\
1 & -\frac{1}{2}+\frac{\sqrt{5}}{2} & 0 \\
1 & 0 & 1 \\
0 & 1 & 1 \\
1 & \frac{1}{2}+\frac{\sqrt{5}}{2} & 1 \\
1 & 1 & \frac{3}{2}-\frac{\sqrt{5}}{2} \\
1 & -\frac{1}{2}+\frac{\sqrt{5}}{2} & -\frac{1}{2}+\frac{\sqrt{5}}{2} \\
1 & 0 & 0 \\
0 & 1 & 0 \\
0 & 0 & 1
\end{array}\right]
$$

It is not difficult to verify that the 3 -cycles of $S_{2}$ correspond to the subsets of 3 collinear points in Figure 3 (the details of a computer implementation are given in an appendix, available on line at http://www.aimath.org/ skrantz/Blurbs/leslie-app.pdf). There are twenty-five 3-cycles, one from each of the five lines with 3 points and four from each of the five lines with 4 points. The 4 -cycles are all sets of 4 points that do not contain a 3-cycle. Each line with 3 points excludes eight 4-cycles. Each subset of three points of a line with 4 points excludes seven 4-cycles and the entire line is also excluded, so a line of four points excludes twenty-nine 4-cycles. Thus there are $330-(8)(5)-(29)(5)=145$ 4-cycles, and 170 cycles of $S_{2}$.

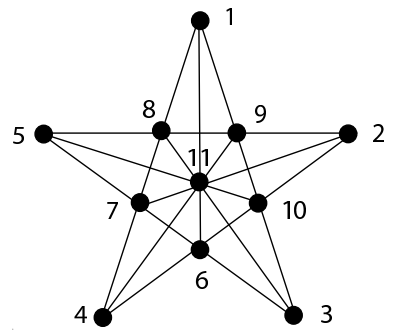

Figure 4: Diagram for $S_{2}$

Theorem 2.6. There is no rational matrix $T$ such that $C_{T}=C_{S_{2}}$.

Proof. The proof is much like that of Theorem 2.3, so we only summarize the steps.

Suppose to the contrary that $W$ is an 11 by $\ell$ matrix of rank $\ell$ over $\mathbb{Q}$ whose cycles are those of $S_{2}$. Since each set of 4 rows of $S_{2}$ is linearly dependent, and $W$ has the same cycles as $S_{2}$, each set of 4 rows of $W$ is linearly dependent. Thus $\ell \leq 3$. Since $\{9,10,11\}$ contains no cycle of $S_{2}$, rows 9,10 and 11 of $W$ form a linearly independent set. Hence $\ell=3$.

By post-multiplying $W$ by an invertible, rational matrix, without loss of generality, we may assume that $W[\{9,10,11\},:]=I_{3}$. 
Since $\{1,9,10\},\{4,9,11\},\{3,9,10\}$ are cycles of $S_{2}$, we may assume (after possibly scaling rows and columns) that row

$$
W[\{1,3,4,9,10,11\},:]=\left[\begin{array}{ccc}
1 & 1 & 0 \\
1 & a & 0 \\
1 & 0 & 1 \\
1 & 0 & 0 \\
0 & 1 & 0 \\
0 & 0 & 1
\end{array}\right] .
$$

Since $\{4,6,10\}$ and $\{1,6,11\}$ are cycles, row 6 of $W$ is (without loss of generality)

$$
\left[\begin{array}{lll}
1 & 1 & 1
\end{array}\right] \text {. }
$$

Since $\{5,10,11\}$ is a cycle of $S_{2}$, row 5 of $W$ has the form

$$
\left[\begin{array}{lll}
0 & 1 & b
\end{array}\right]
$$

for some nonzero $b$. Using the cycles $\{2,5,9\}$ and $\{2,4,10\}$, we see that row 2 is

$$
\left[\begin{array}{lll}
1 & 1 / b & 1
\end{array}\right] \text {. }
$$

Because $\{2,7,11\}$ and $\{1,4,7\}$ are cycles, row 7 of $A$ has the form

$$
\left[\begin{array}{lll}
1 & 1 / b & 1-1 / b
\end{array}\right] \text {. }
$$

Since $\{3,5,7\}$ is a cycle, $0=\operatorname{det} A[\{3,5,7\},:]=a b-1 / b$, so $a b=1 / b$. Similarly, $0=\operatorname{det} A[\{3,5,6\},:]=1+a b-b$, and substitution of $a b=1 / b$ into this equation yields the equation $1+1 / b-b=0$. Thus, $b=\frac{1 \pm \sqrt{5}}{2}, b$ is irrational, and we have obtained a contradiction.

The proof of the next corollary is virtually identical to that of Corollary 2.4, and is left to the reader.

Corollary 2.7. $\operatorname{mr}\left(\mathcal{M}_{C_{S_{2}}}^{\mathbb{Q}}\right)=9>8=\operatorname{mr}\left(\mathcal{M}_{C_{S_{2}}}^{\mathbb{R}}\right)$.

Corollary 2.7 provides a counterexample to the central conjecture in [AHKLR, pp. 112-113].

In this paper we raise the following basic conjecture. For any $m \times n$ sign pattern matrix $A$ with $\operatorname{mr}(A)=k$, there exists a rational matrix (equivalently, an integer matrix) $B \in \mathcal{Q}(A)$ such that rank $B=k$.

With our notation, this would be:

For any $m \times n$ sign pattern matrix $Z$ with $\operatorname{mr}\left(\mathcal{M}_{Z}^{\mathbb{R}}\right)=k$, there exists a rational matrix (equivalently, an integer matrix) $B$ in the sign pattern class of $Z$ such that rank $B=k$.

The sign-pattern class restricts the signs of the entries, a stronger restriction than restricting the zero-nonzero pattern. Thus we have 
Counterexample 2.8. Let $A$ be a realization of $C_{S_{2}}^{\mathbb{R}}$ of rank 8 , and let $Z_{C_{S_{2}}}$ be the sign pattern of $A$. By Corollary 2.7 there is no rational matrix with sign pattern $Z$ of rank 8 . Hence the minimum rank among the rational matrices with sign pattern $Z$ is larger than the minimum rank among the real matrices with sign pattern $Z_{C_{S_{2}}}$. An explicit example of such $Z_{C_{S_{2}}}$ and details of its construction are given in an appendix, available on line at http://www.aimath.org/ skrantz/Blurbs/leslie-app.pdf. (After the submission of this paper, the authors became aware of another sign pattern $A$, for which $\mathrm{mr}^{\mathbb{Q}}(A)>\mathrm{mr}^{\mathbb{R}}(A)$, that was presented in $[\mathrm{KR}]$.)

Note that in the proof of Theorem 2.6, row 8 of $S_{2}$ was not used. We conclude that there is no rational matrix whose cycle matrix is $S_{2}[\overline{\{8\}},:]$. As there is an automorphism of Figure 1 that takes 8 to any one of $\{1,2, \ldots, 10\}$, we can replace 8 by any one of $\{1,2, \ldots, 10\}$. Just like Corollary 2.5 , we have the following result, whose proof is left to the reader.

Corollary 2.9. Let $S$ be a pattern obtained from $S_{2}$ be deleting any one of rows $1, \ldots, 10$. Then

$$
\operatorname{mr}\left(C_{S}^{\mathbb{Q}}\right)=8>7=\operatorname{mr}\left(C_{S}^{R}\right) .
$$

\section{Graphs and minimum rank}

We now return to the question of variation over $\mathbb{F}=\mathbb{C}, \mathbb{R}$, or $\mathbb{Q}$ of $\operatorname{mr}\left(\mathcal{S}_{G}^{\mathbb{F}}\right)$, the minimum rank of a graph over $\mathbb{F}$. Recall that the matrices in $\mathcal{S}_{G}^{\mathbb{F}}$ are symmetric and the diagonal is unrestricted.

Let $C_{S_{1}}$ be a cycle matrix of $S_{1}$, and let $G_{1}$ be the bipartite graph whose bi-adjacency matrix is $C_{S_{1}}$. Thus, $G_{1}$ has 9 vertices, say $1,2, \ldots, 9$, corresponding to the columns of $C_{S_{1}}$ and 66 vertices corresponding to the rows of $C_{S_{1}}$, for a total of 75 vertices.

Note that if $M$ is a minimal rank realization of $\mathcal{M}_{C_{S_{1}}}^{\mathbb{C}}$, respectively, $\mathcal{M}_{C_{S_{1}}}^{\mathbb{R}}$, then

$$
\left[\begin{array}{c|c}
O & M^{T} \\
\hline M & O
\end{array}\right]
$$

is a complex (respectively real) matrix of rank $6+6=12$ (respectively, $7+7=14$ ) whose graph is $G_{1}$. Hence, $\operatorname{mr}\left(\mathcal{S}_{G_{1}}^{\mathbb{C}}\right) \leq 12$ and $\operatorname{mr}\left(\mathcal{S}_{G_{1}}^{\mathbb{R}}\right) \leq 14$. We claim that equality holds in both of these inequalities.

Theorem 3.1. $\operatorname{mr}\left(\mathcal{S}_{G_{1}}^{\mathbb{R}}\right)=14>12=\operatorname{mr}\left(\mathcal{S}_{G_{1}}^{\mathbb{C}}\right)$.

Proof. Let $A$ be a matrix whose graph is $G_{1}$. Thus, $A$ has the form

$$
\left[\begin{array}{c|c}
D & B^{T} \\
\hline B & E
\end{array}\right]
$$

where $D$ and $E$ are diagonal matrices, and $B$ has pattern $C_{S_{1}}$. We claim that if $A$ is complex (respectively real), then $\operatorname{rank}(A) \geq 12$ (respectively, $\operatorname{rank}(A) \geq 14)$ 
If each diagonal entry of $E$ is 0 and $A$ is complex (respectively, real), then by Corollary $2.4, \operatorname{rank}(A) \geq \operatorname{rank}(B)+\operatorname{rank}\left(B^{T}\right) \geq 6+6=12$ (respectively, $\operatorname{rank}(A) \geq \operatorname{rank}(B)+$ $\left.\operatorname{rank}\left(B^{T}\right) \geq 7+7=14\right)$.

If $A$ is complex (respectively, real) and $E$ has 12 (respectively 14) or more nonzero entries, then $\operatorname{rank}(A) \geq \operatorname{rank}(E) \geq 12$ (respectively, $\operatorname{rank}(A) \geq \operatorname{rank}(E) \geq 14$ ). Otherwise, $A$ is complex (respectively, real) and $E$ has $k$ nonzero entries with $1 \leq k \leq 11$ (respectively, $1 \leq k \leq 13$ ).

Observe that rows 1,2 and 4 of $S_{1}$ are linearly independent. Therefore, for each $j \in\{1,2, \ldots, 9\} \backslash\{1,2,4\}$ there is a unique cycle of $S_{1}$ that contains $j$ and is contained in $\{1,2,4, j\}$. It can be verified that these cycles are

$$
\{1,2,3\},\{1,2,4,5\},\{1,2,4,6\},\{1,4,7\},\{1,2,4,8\},\{2,4,9\} .
$$

Let $\alpha_{1}$ be the indices of the rows of $B$ corresponding to the these cycles. Similarly, let $\alpha_{2}$ the indices of the rows corresponding to the cycles

$$
\{6,8,1\},\{5,8,2\},\{5,6,8,3\},\{5,6,4\},\{5,6,8,7\},\{5,6,8,9\},
$$

determined by linearly independent rows $5,6,8$ (the order in which the entries in a cycle are listed is irrelevant, and we have listed all the entries of the cycle that are in 5,6,8 first). Let $\alpha_{3}$ the indices of the rows corresponding to

$$
\{3,7,9,1\},\{3,7,9,2\},\{3,7,9,4\},\{3,7,5\},\{3,9,6\},\{7,9,8\},
$$

determined by the linearly independent rows $3,7,9$. Note that the $\alpha_{\ell}$ are mutually disjoint. By construction (cf. Lemma 2.1), each $C_{S_{1}}\left[\alpha_{\ell},:\right]$ has a 6 by 6 permutation matrix as a submatrix.

Let $\beta=\left\{j: e_{j j} \neq 0\right\}$. By the Pigeonhole Principle, there is a $j$ such that $\left|\alpha_{j} \cap \beta\right| \leq$ $\lfloor k / 3\rfloor$. Thus, $A\left[\alpha_{j} \cup \beta\right]$ is permutation similar to a matrix of the form

$\left[\begin{array}{c|c|c} & B\left[\alpha_{j} \backslash \beta,:\right]^{T} & \\ \hline B\left[\alpha_{j} \backslash \beta,:\right] & O & O \\ \hline & O & E[\beta]\end{array}\right]$,

and thus has rank at least $k+2(6-\lfloor k / 3\rfloor) \geq 12+\frac{1}{3} k>12$. Hence, if $A$ is complex, then $\operatorname{rank}(A) \geq 12$, and it follows that $\operatorname{mr}\left(\mathcal{S}_{G_{1}}^{\mathbb{C}}\right)=12$.

Otherwise, $A$ is real and

$$
\operatorname{rank}(A) \geq 12+k-2\lfloor k / 3\rfloor
$$

Hence, $\operatorname{rank}(A) \geq 14$, except in possibly the cases that $k=1$ or $k=3$. Note that even in these cases, we have already proved that $\operatorname{rank}(A) \geq 13$ and thus that $\operatorname{mr}\left(\mathcal{S}_{G_{1}}^{\mathbb{R}}\right) \geq 13>$ $12=\operatorname{mr}\left(\mathcal{S}_{G_{1}}^{\mathbb{C}}\right)$.

First consider the case that $k=1$. Without loss of generality, $e_{11}=1$. Let $\alpha$ be the cycle of $S_{1}$ corresponding to row 1 of $B$, and let $j \in \alpha$. Let $\tau=\left\{\ell: b_{\ell, j}=0\right\}$, and observe 
the $B[\tau, \overline{\{j\}}]$ is a realization of the cycle matrix obtained from $S_{1}$ by deleting the $j$ th row. Thus, by Corollary $2.5, B[\tau, \overline{\{j\}}]$ has rank at least 6 . Since $j$ appears in a cycle other than $\alpha$, it follows that $M$ has a submatrix of the form

$\left[\begin{array}{c|c|c|c|c} & & & & B[\tau, \overline{\{j\}}]^{T} \\ \hline & & & b & 0 \cdots 0 \\ \hline & & 1 & 0 & 0 \cdots 0 \\ \hline & b & 0 & 0 & 0 \cdots 0 \\ \hline \multirow{3}{*}[\tau,\overline{\{j\}}]{} & 0 & 0 & 0 & \\ & \vdots & \vdots & \vdots & O \\ & 0 & 0 & 0 & \end{array}\right]$,

with $b \neq 0$, and we conclude that $A$ has rank at least $6+3+6=15>14$.

Next consider the case $k=3$. Assume to the contrary that $M$ has rank 13 . Inequality (2) implies that $\left|\alpha_{j} \cap \beta\right|=1$ for $j=1,2,3$; otherwise rank $A \geq \operatorname{rank} A\left[\alpha_{j} \cup \beta\right] \geq 12+k=15$ for some $j$. The affine plane $\operatorname{AG}(2,3)$ has 4 sets of parallel lines. Since $|\beta|=3$, there exist two non-parallel lines of $\operatorname{AG}(2,3)$ neither of which corresponds to a row of $B$ whose index is in $\beta$. Without loss of generality, we may assume that these lines are $\{1,2,3\}$ and $\{2,4,9\}$.

Now let

$$
\begin{aligned}
& \alpha_{1}^{\prime}=\{\{1,2,3\},\{2,9,4\},\{1,9,5\},\{1,2,9,6\},\{1,2,9,7\},\{1,2,9,8\}\}, \\
& \alpha_{2}^{\prime}=\{\{3,4,5,1\},\{3,4,5,2\},\{4,5,6\},\{3,5,7\},\{3,4,8\},\{3,4,5,9\}\}, \\
& \alpha_{3}^{\prime}=\{\{6,8,1\},\{6,7,2\},\{6,7,8,3\},\{6,7,8,4\},\{6,7,8,5\},\{7,8,9\}\} .
\end{aligned}
$$

It is easy to verify that the $\alpha_{j}^{\prime}$ are mutually disjoint sets of cycles of $S_{1}$. Hence, arguing as before, $\left|\alpha_{j}^{\prime} \cap \beta\right|=1$ for each $\alpha_{j}^{\prime}$. Note that $\alpha_{1}^{\prime}$ and $\alpha_{2}$ and $\alpha_{3}$ are mutually disjoint, and $\alpha_{1} \cap \alpha_{1}^{\prime}=\{\{1,2,3\},\{2,4,9\}\}$. Hence, $\beta$ contains an index that corresponds to either $\{1,2,3\}$ or $\{2,4,9\}$, which is a contradiction. Hence, $A$ has rank at least 14 , as desired.

Let $C_{S_{2}}$ be a cycle matrix of $S_{2}$, and let $G_{2}$ be the bipartite graph whose bi-adjacency matrix is $M$. Thus, $G_{2}$ has 11 vertices, say $1,2, \ldots, 11$, corresponding to the columns of $C_{S_{2}}$ and 170 additional vertices corresponding to the rows of $C_{S_{2}}$ (and hence to the cycles of $\left.S_{2}\right)$, for a total of 181 vertices. As with the real vs. complex case, one can see immediately that $\operatorname{mr}\left(\mathcal{S}_{C_{S_{2}}}^{\mathbb{R}}\right) \leq 16$ and $\operatorname{mr}\left(\mathcal{S}_{C_{S_{2}}}^{\mathbb{Q}}\right) \leq 18$. We claim that equality holds in both of these inequalities.

Theorem 3.2. $\operatorname{mr}\left(\mathcal{S}_{G_{2}}^{\mathbb{Q}}\right)=18>16=\operatorname{mr}\left(\mathcal{S}_{G_{2}}^{\mathbb{R}}\right)$.

Proof. The proof proceeds as that of Theorem 3.1. Let $A$ be a matrix whose graph is $G_{2}$. Thus, $A$ has the form (1) where $D$ and $E$ are diagonal matrices, and $B$ has pattern $C_{S_{2}}$. We claim that if $A$ is real (respectively rational), then rank $A \geq 16$ (respectively, $\operatorname{rank} A \geq 18$ ) 
As before, the cases $E$ has 0 or at least 16 (or 18 in the rational case) nonzero entries is easily handled. Otherwise, $A$ is real (respectively, rational) and $E$ has $k$ nonzero entries with $1 \leq k \leq 16$ (respectively, $1 \leq k \leq 18$ ).

Now choose five disjoint 3-sets of independent rows of $S_{2}$ (non-cycle 3-sets)in such a way as to produce five pairwise disjoint sets of eight cycles. Specifically, for the independent sets we can use $\{1,2,6\},\{2,3,7\},\{3,4,8\},\{4,5,9\},\{1,5,10\}$, yielding the following five sets of eight cycles:

$$
\begin{aligned}
\alpha_{1}= & \{\{1,2,6,3\},\{2,6,4\},\{1,2,6,5\},\{1,2,6,7\},\{1,2,6,8\},\{1,2,6,9\},\{2,6,10\},\{1,6,11\}\} \\
\alpha_{2}= & \{\{2,3,7,1\},\{2,3,7,4\},\{3,7,5\},\{3,7,6\},\{2,3,7,8\},\{2,3,7,9\},\{2,3,7,10\},\{2,7,11\}\} \\
\alpha_{3}= & \{\{4,8,1\},\{3,4,8,2\},\{3,4,8,5\},\{3,4,8,6\},\{4,8,7\},\{3,4,8,9\},\{3,4,8,10\},\{3,8,11\}\} \\
\alpha_{4}= & \{\{4,5,9,1\},\{5,2,9\},\{4,5,3,9\},\{4,5,9,10\},\{4,5,9,6\},\{4,5,9,7\},\{5,9,8\},\{4,9,11\}\} \\
\alpha_{5}= & \{\{1,5,10,2\},\{1,10,3\},\{1,5,10,4\},\{1,5,10,6\},\{1,5,10,7\},\{1,5,10,8\},\{1,10,9\}, \\
& \{5,10,11\}\}
\end{aligned}
$$

These comprise disjoint sets of 8 cycles of $S_{2}$ and hence $B\left[\alpha_{j},:\right]$ contains a 8 by 8 permutation matrix for each $j$.

Arguing as in the proof of Theorem 3.1, we see that there is a $j$ such that $\left|\alpha_{j} \cap \beta\right| \leq$ $\lfloor k / 5\rfloor$. Thus, $A\left[\alpha_{j} \cup \beta\right]$ is a matrix of the form

$\left[\begin{array}{c|c|c} & B[\alpha \backslash \beta,:]^{T} & \\ \hline B[\alpha \backslash \beta,:] & O & O \\ \hline & O & E[\beta]\end{array}\right]$,

and has rank at least $k+2(8-\lfloor k / 5\rfloor) \geq 16+3 k / 5>16$. Hence, if $A$ is real, then $\operatorname{rank}(A) \geq 16$, and it follows that $\operatorname{mr}\left(\mathcal{S}_{G_{2}}^{\mathbb{R}}\right)=16$.

Otherwise, $A$ is rational and

$$
\operatorname{rank}(A) \geq 16+k-2\lfloor k / 5\rfloor
$$

Hence, $\operatorname{rank}(A) \geq 18$, except possibly in the case that $k=1$. This case is handled just as in the proof of Theorem 3.1. Hence, $A$ has rank at least 18, as desired.

\section{Minimum rank and extension fields}

Returning now to a not-necessarily symmetric pattern $Z$ with the diagonal restricted by the pattern, it is natural to ask for the relationship between $\operatorname{mr}\left(\mathcal{M}_{Z}^{E}\right)$ and $\operatorname{mr}\left(\mathcal{M}_{Z}^{F}\right)$, in the case that $E$ is an extension field of $F$. It is clear that $\operatorname{mr}\left(\mathcal{M}_{Z}^{E}\right) \leq \operatorname{mr}\left(\mathcal{M}_{Z}^{F}\right)$. If $E$ is an extension field of $F$, then there exists an $F$-vector space $C$ such that $E$ is the direct sum of the $F$-vector spaces $F$ and $C$. Thus, each $e \in E$ can be uniquely expressed as $e=f+c$ where $f \in F$ and $c \in C$. We call $f$ the $F$-component of $e$.

Theorem 4.1. Let $E$ and $F$ be fields with $|E: F|=d<\infty$ and let $Z$ be an $m$ by $n$ pattern with $|F|>n$. Then $\operatorname{mr}\left(\mathcal{M}_{Z}^{F}\right) \leq d \cdot \operatorname{mr}\left(\mathcal{M}_{Z}^{E}\right)$. 
Proof. Let $A$ be realization of $Z$ over $E$. We claim that there exists a diagonal matrix $D$ over $E$ such that the first $F$-component of each nonzero entry of $A D$ is nonzero. This is clear if $|E|=\infty$. Otherwise, for each nonzero element $x$ of $E$ there are at most $|F|^{d-1}$ elements $e$ of $E$ such that the first $F$-component of $e x$ is 0 . Thus, for each column of $A$ there are at most $n|F|^{d-1}$ elements $e$ of $E$ such that scaling that column by $e$ results in a column with at least one nonzero entry whose first $F$-component is 0 . Since $n|F|^{d-1}<|E|$, there exists an invertible diagonal matrix $D$ such that each nonzero entry of $A D$ has a nonzero first component.

Without loss of generality, we may take $D=I$. Let $1=\alpha_{1}, \alpha_{2}, \ldots, \alpha_{d}$ be a basis of $E$ viewed as an $F$-vector space. Let $B_{1}, \ldots, B_{d}$ be the unique matrices over $F$ such that

$$
A=B_{1}+\alpha_{2} B_{2}+\cdots \alpha_{d} B_{d}
$$

Since $D=I, B_{1}$ is a realization of $Z$ over $F$.

Let $V$ be the column space of $A$. Let $v_{1}, v_{2}, \ldots, v_{k}$ be a basis of $V$ viewed as an $E$-vector space. Note that $V$ may also be viewed as a $F$ vector space. Moreover $V$ as an $F$ vector space has spanning set $\alpha_{j} v_{\ell}(1 \leq j \leq d, 1 \leq \ell \leq k)$. Hence, the $\operatorname{dim}^{F}(V) \leq d \cdot \operatorname{dim}^{E}(V)$.

Note that $\left\{B_{1} x+\alpha_{2} B_{2} x+\cdots+\alpha_{d} B_{d} x: x \in F^{n}\right\}$ is a subspace contained in the $F$-vector space $V$, and clearly has dimension at least $\operatorname{rank}\left(B_{1}\right)$. Hence, $\operatorname{rank}\left(B_{1}\right) \leq d \cdot \operatorname{rank}(A)$, and the result follows.

Thus, $\frac{\operatorname{mr}\left(\mathcal{M}_{Z}^{\mathbb{R}}\right)}{\operatorname{mr}\left(\mathcal{M}_{Z}^{\mathbb{C}}\right)} \leq 2$ for all patterns $M$ and $\frac{\operatorname{mr}\left(\mathcal{M}_{C_{S}}^{\mathbb{R}}\right)}{\operatorname{mr}\left(\mathcal{M}_{C_{S}}^{\mathbb{C}}\right)} \geq \frac{6}{5}$ where $C_{S}$ is the pattern in Corollary 2.5. Two questions arise:

1. What is the supremum of $\frac{\operatorname{mr}\left(\mathcal{M}_{Z}^{\mathbb{R}}\right)}{\operatorname{mr}\left(\mathcal{M}_{Z}^{\mathbb{C}}\right)}$ ?

2. Is there an upper bound on $\frac{\operatorname{mr}\left(\mathcal{M}_{Z}^{\mathbb{Q}}\right)}{\operatorname{mr}\left(\mathcal{M}_{Z}^{\mathbb{R}}\right)}$ ?

\section{Computation of minimum rank}

The question of the decidability of the minimum rank of a graph over a field $F$ was raised at the 2006 American Institute of Mathematics workshop, "Spectra of Families of Matrices described by Graphs, Digraphs, and Sign Patterns," and in this section we briefly discuss theoretical algorithms for the computation of minimum rank, their complexity, and some implications in the cases that $F$ is the real or complex field.

A conversion of the problem of computing the minimal rank of a graph $G$ with vertices $1,2, \ldots, n$ and edge-set $E$ over $F$ to verifying the validity or invalidity of statements over $F$ is given by the following well-known equivalent statements:

(a) $\operatorname{mr}^{F}(G) \leq k$. 
(b) There exist $x^{(1)}, \ldots, x^{(k)}, y^{(1)}, \ldots, y^{(k)} \in F^{n}$ such that

$$
\bigwedge_{i=1}^{n} \bigwedge_{j>i}\left[\left(b_{i j}-b_{j i}=0\right) \wedge\left(b_{i j} \neq 0 \text { if } i j \in E, \text { and } b_{i j}=0 \text { if } i j \in \Delta\right)\right]
$$

where $b_{i j}=\left(\sum_{t=1}^{k} x^{(t)}\left(y^{(t)}\right)^{T}\right)_{i j}$.

(c) There exist $b_{i i} \in F, i=1, \ldots, n$, and $b_{i j} \in F$ for $i<j, i j \in E$ such that

$$
\bigwedge_{i j \in E} b_{i j} \neq 0 \bigwedge_{\alpha, \beta \subseteq\langle n\rangle,|\alpha|=|\beta|=k+1} \operatorname{det} B[\alpha, \beta]=0
$$

where $b_{i j}=0$ for $i j \in \Delta, b_{j i}=b_{i j}$ and $B=\left[b_{i j}\right]$.

(d) There exist $b_{i i} \in F, i=1, \ldots, n$, and $b_{i j} \in F$ for $i<j, i j \in E$ such that

$$
\bigwedge_{i j \in E} b_{i j} \neq 0 \bigwedge \bigwedge_{\alpha \subseteq\langle n\rangle,|\alpha| \geq k+1} \operatorname{det} B[\alpha, \alpha]=0
$$

where $b_{i j}=0$ for $i j \in \Delta, b_{j i}=b_{i j}$ and $B=\left[b_{i j}\right]$.

Here $\Delta$ denotes the set of ordered pairs $(i, j)$ of integers with $i \neq j, 1 \leq i, j \leq n$, and $i j \notin E$. Note that an inequation, $f\left(z_{1}, \ldots, z_{\ell}\right) \neq 0$, can be made into an equation $y f\left(z_{1}, x_{2}, \ldots, z_{\ell}\right)=1$ by introducing a new variable $y$. Also, note in the case that $F=\mathbb{R}$, the statement

(e) There exist $x^{(1)}, \ldots, x^{(k)} \in F^{n}, \lambda_{1}, \ldots, \lambda_{k} \in F$ such that

$$
\bigwedge_{i=1}^{n} \bigwedge_{j>i}\left[\left(b_{i j}-b_{j i}=0\right) \wedge\left(b_{i j} \neq 0 \text { if } i j \in E, \text { and } b_{i j}=0 \text { if } i j \in \Delta\right)\right]
$$

where $b_{i j}=\left(\sum_{t=1}^{k} \lambda_{t} x^{(t)}\left(x^{(t)}\right)^{T}\right)_{i j}$.

is equivalent to each of the statements (a)-(d).

Quantifier elimination (when available) allows one to verify the validity of statements of the form that appear in (a)-(e). Over the complex numbers, the insolvability of a system of polynomial equations and inequations is determined by Hilbert's Nullstellensatz. It says that a system of polynomials is unsolvable if and only if a certain ideal contains the constant function 1. So the problem is reduced to finding a good basis for a given ideal. This can be done efficiently by finding a Gröbner basis, and this provides a theoretical algorithm for determining $\mathrm{mr}^{\mathbb{C}}(G)$ for any graph $G$.

Tarski $[\mathrm{T}]$ was the first to observe that quantifier-elimination can also be done over every real closed field; in fact, Tarski produced an algorithm that does it. Algorithms have been improved over the years and software for verifying the validity of sentences (that are not too long) over the real or complex numbers is available. 
An algorithm by Renegar $[\mathrm{R}]$ provides improved complexity bounds over the real numbers, and needs at most $(M d)^{O(1) N}$ steps, where $M$ is the number of equations and inequations, $N$ is the number of variables, and $d$ is the maximum degree of the polynomials involved. We refer the readers to the recent paper $[\mathrm{BR}]$ on applications of the Renegar algorithm. Improved complexity bounds for the Renegar algorithm are available when executed on parallel processors.

Some computer algebra systems, such as Mathematica, have implemented quantifier elimination, and Jason Grout [G] has developed a Mathematica notebook to compute the minimum rank of very small graphs over $\mathbb{R}$ or $\mathbb{C}$ by verifying the validity of statements in (b)-(e).

Table 1 lists the values of the corresponding parameters $M, d$ and $N$ for the characterizations of minimum rank $k$ given by conditions (b)-(e):

Table 1: Values of parameters $M, d, N$

\begin{tabular}{|c|c|c|c|}
\hline & $M$ & $d$ & $N$ \\
\hline$(b)$ & $n(n-1)$ & 2 & $2 n k$ \\
\hline $\begin{array}{c}(c) \\
(d)\end{array}$ & $\left.|E|+\sum_{r=k+1}^{n} \begin{array}{c}n \\
k+1\end{array}\right)^{2}$ \\
$\left(\begin{array}{l}n \\
r\end{array}\right)$ & $n$ & $|E|+n$ \\
\hline$(e)$ & $n(n-1)$ & 3 & $(n+1) k$ \\
\hline
\end{tabular}

Tarski's decidability theorem does not apply to decisions over the field of rationals. We note that the decidability problem of the minimum rank of a graph over the rationals is still open. 


\section{References}

[AHKLR] M. Arav, F. Hall, S. Koyucu, Z. Li and B. Rao. On Rational Realizations of the Minimums Rank of a Sign Pattern Matrix. Linear Alg. Appls., 2005, 409:11-125.

[BFH] F. Barioli, S.M. Fallat, and L. Hogben. Computation of minimal rank and path cover number for graphs. Linear Alg. Appls., 2004, 392:289-303.

[BHL] W. Barrett, H. van der Holst and R. Loewy. Graphs whose minimal rank is two. Electronic Journal of Linear Algebra, 2004, 11:258-280.

[BR] A. Berman and U. Rothblum. A note on the computation of the CP-rank, Linear Algebra Appl. 2006, 419:1-7.

[FH] S.M. Fallat and L. Hogben. The Minimum Rank of Symmetric Matrices Described by a Graph: A Survey. Linear Alg. Appls., 2007, 426:558-582.

[F] M. Fiedler. A characterization of tridiagonal matrices. Linear Alg. Appls., 1969 2:191-197.

[G] J. Grout. Minimum rank calculation using Mathematica. Software available from the author (grout@iastate.edu).

[HH] L. Hogben and H. van der Holst. Forbidden minors for the class of graphs $G$ with $\xi(G) \leq 2$. Linear Alg. Appls., 2007, 423: 42-52. .

[JLS] C. R. Johnson, R. Loewy and P. A. Smith. The graphs for which maximum multiplicity of an Eigenvalue is Two. Preprint. Available at http://arxiv.org/pdf/math.CO/0701562.

[KR] Swastik Kopparty, K. P. S. Bhaskara Rao.The Minimum Rank Problem: a counterexample. Presentation at the 14th International Linear Algebra Society Conference, Shanghai, China, July 16, 2007.

[M] Saunders MacLane, Some Interpretations of Abstract Linear Dependence in terms of Projective Geometry, Amer. J. of Mathematics, 1936, 58:236-240.

[O] J. G. Oxley, Matroid Theory, Oxford University Press, Oxford, 1992.

[R] J. Renegar, On the computation complexity and geometry of the first-order theory of the reals, parts I,II,III, J. Symbolic Logic, 1992, 13:255-352.

[RS] W.C. Rheinboldt and R.A. Shepherd. On a characterization of tridiagonal matrices by M. Fiedler. Linear Alg. Appls., 1974, 8:87-90.

[T] A. Tarski, A Decision Method for Elementary Algebra and Geometry, 2nd ed. rev., University of California Press, Berkeley, 1951. 